\title{
PROPRIEDADES FÍSICAS DE FILMES BIODEGRADÁVEIS À BASE DE AMIDO DE MANDIOCA, ÁLCOOL POLIVINÍLICO E MONTMORILONITA
}

\author{
Fernando de Oliveira Faria, Ana Elisa Stefani Vercelheze e Suzana Mali* \\ Departamento de Bioquímica e Biotecnologia, Centro de Ciências Exatas, Universidade Estadual de Londrina, CP 6001, \\ 86051-990 Londrina - PR, Brasil
}

Recebido em 15/4/11; aceito em 5/9/11; publicado na web em 26/9/11

\begin{abstract}
PHYSICAL PROPERTIES OF BIODEGRADABLE FILMS BASED ON CASSAVA STARCH, POLYVINYL ALCOHOL AND MONTMORILLONITE. The objective of this work was to manufacture biodegradable films based on cassava starch, polyvinyl alcohol (PVA) and sodium montmorillonite (Na-MMT), using glycerol as a plasticizer. These films were characterized according to their microstructure, optical, mechanical, and barrier properties. The combination of starch-PVA-MMT resulted in films with a more homogeneous surface than starch films. The introduction of PVA into the starch matrix led to the formation of films with lower water vapor permeability (WVP), higher tensile strength and greater elongation. MMT was exfoliated in the films, resulting in greater stability for different relative humidities, lower WVP, higher resistance and lower flexibility.
\end{abstract}

Keywords: microstructure; mechanical properties; sodium montmorillonite.

\section{INTRODUÇÃO}

Os filmes biodegradáveis compõem parte importante do cenário de pesquisa nacional e internacional, com trabalhos importantes quanto à caracterização, formulação e aplicação destes materiais. Existem grandes possibilidades de utilização para os sistemas de embalagens biodegradáveis à base de amido, em especial na área de alimentos, devido ao seu baixo custo e abundância, no entanto, a produção de embalagens exclusivamente de amido não é viável devido à sua higroscopicidade e baixa flexibilidade, o que torna estes materiais sensíveis ao contato direto com a água e quebradiços. ${ }^{1}$

Com a finalidade de melhorar estas características, vários trabalhos têm sido propostos nos últimos $\operatorname{anos}^{2-5} \mathrm{e}$, neste sentido, a incorporação de cargas minerais nanométricas ${ }^{6-9}$ e a mistura de mais de outros polímeros biodegradáveis, como o álcool polivinílico (PVA), podem ser consideradas alternativas interessantes, pois além de manter a biodegradabilidade, atuam como reforço mecânico e podem diminuir a permeabilidade destes materiais ao vapor de água.

O PVA é um polímero sintético, hidrofílico, biodegradável, não tóxico, biocompatível e que apresenta boa capacidade de formação de filmes. Devido sua alta compatibilidade com a matriz polimérica de amido e as suas características peculiares, tais como, propriedade de adesão, elevada resistência mecânica, barreira ao oxigênio, aromas, óleos e solventes, o PVA é o polímero sintético bastante promissor para a produção de embalagens biodegradáveis, deixando-as tão resistentes quanto as embalagens convencionais. ${ }^{10-13}$

As nanoargilas, compostas predominantemente pelo argilomineral montmorilonita (55-70\%), do grupo das esmectitas, são conhecidas pelo nome genérico de bentonitas. A montmorilonita (MMT) possui partículas com tamanhos que podem variar de 2-0,1 $\mu \mathrm{m}$, podendo apresentar formato de placas ou lâminas. Pertence ao grupo dos filossilicatos, cujas placas são caracterizadas por unidades estruturais constituídas por uma folha de tetraedros de sílica, ligados pelos oxigênios localizados nos vértices da base, e uma folha de octaedros de alumina, ligados pelas faces laterais. ${ }^{14}$ As camadas de montmorilonita possuem espessura inferior ao comprimento de onda de luz visível, não desviam a luz, sendo então transparentes, resistentes a solventes e a altas temperaturas; estas características são interessantes nos processos de transformação ou reciclagem, como também características desejáveis para a produção de nanocompósitos para aplicação em embalagens. ${ }^{15}$

$\mathrm{Na}$ literatura são relatados vários métodos de preparação de misturas polímero-montmorilonita, visando a obtenção de nanocompósitos. Esses métodos dependem da natureza da argila e do polímero. Entre eles pode-se citar: polimerização in situ, intercalação de polímero fundido ou melt processing, método sol-gel e dispersão aquosa. A dispersão aquosa consiste em um sistema bifásico, no qual a montmorilonita é dispersa na fase aquosa e misturada ao polímero sob aquecimento. ${ }^{16}$ Este último método tem sido descrito como método apropriado para a mistura de amido e nanoargila, devido às características hidrofílicas do sistema, ${ }^{7,8,17}$ sendo este o método empregado neste trabalho.

A interação amido/argila é favorecida quando a estrutura é completamente esfoliada, isto é, quando existe um grande espaçamento entre as camadas da MMT, o que aumenta significativamente a área superficial de contato da argila, resultando em melhores propriedades mecânicas e de barreira para os nanocompósitos produzidos. ${ }^{18}$

Diante disto, os objetivos do presente trabalho foram produzir filmes biodegradáveis à base de misturas de amido/álcool polivinílico/glicerol, com adição de nanoargilas e, ainda, caracterizar estes materiais quanto às suas propriedades mecânicas, ópticas, de barreira e microestrutura.

\section{PARTE EXPERIMENTAL}

\section{Material}

Foi utilizado amido de mandioca (Manihot esculenta Crantz) da Indemil Brasil no preparo dos filmes. Glicerol de grau analítico foi adquirido da Synth Brasil, a montmorilonita sódica (MMT-Na) da marca Closite ${ }^{\circledR} \mathrm{Na}^{+}$foi adquirida da Southern Clay Products (Texas - EUA) e álcool polivinílico (PVA) com grau de hidrólise entre 86,5$89,5 \%$ foi adquirido da Reagen. 


\section{Preparo dos filmes}

Os filmes foram produzidos pelo processo de casting, empregando-se água destilada como solvente. As soluções filmogênicas foram preparadas com concentração de $3 \%(\mathrm{p} / \mathrm{v})$ de sólidos, obtidos pela mistura amido, PVA e MMT-Na, com concentrações que variaram conforme descrito na Tabela 1. Foram usadas siglas para designar cada formulação: PVA0, PVA1, PVA2, PVA3 e PVA5 (formulações com adição de PVA nas concentrações de $0,1,2,3$ e 5 g/100 g sólidos, respectivamente), PVA0-MMT2.5, PVA1-MMT2.5, PVA2MMT2.5, PVA3-MMT2.5 e PVA5-MMT2.5 (formulações com adição de PVA nas concentrações de 0,1,2, 3 e 5 g/100 g sólidos, respectivamente, e todas com adição de 2,5 g de MMT por $100 \mathrm{~g} /$ sólidos) e PVA0-MMT5.0, PVA1- MMT5.0, PVA2- MMT5.0, PVA3MMT5.0 e PVA5- MMT5.0 (formulações com adição de PVA nas concentrações de $0,1,2,3$ e $5 \mathrm{~g} / 100 \mathrm{~g}$ sólidos, respectivamente, e todas com adição de 5,0 g de MMT por $100 \mathrm{~g} /$ sólidos). O glicerol foi empregado como plastificante, com concentração fixada em $20 \mathrm{~g}$ de glicerol/100 g de sólidos.

Tabela 1. Quantidades de amido, PVA e montmorilonita em cada formulação

\begin{tabular}{lccc}
\hline Formulações & $\begin{array}{c}\text { Amido } \\
(\mathrm{g} / 100 \mathrm{~g} \text { sólidos })\end{array}$ & $\begin{array}{c}\text { PVA } \\
(\mathrm{g} / 100 \mathrm{~g} \text { sólidos })\end{array}$ & $\begin{array}{c}\text { MMT-Na } \\
(\mathrm{g} / 100 \mathrm{~g} \text { sólidos })\end{array}$ \\
\hline PVA0 & 100,0 & 0 & 0 \\
PVA1 & 99,0 & 1 & 0 \\
PVA2 & 98,0 & 2 & 0 \\
PVA3 & 97,0 & 3 & 0 \\
PVA5 & 95,0 & 5 & 0 \\
PVA0MMT2.5 & 97,5 & 0 & 2,5 \\
PVA1 MMT2.5 & 96,5 & 1 & 2,5 \\
PVA2 MMT2.5 & 95,5 & 2 & 2,5 \\
PVA3 MMT2.5 & 94,5 & 3 & 2,5 \\
PVA5 MMT2.5 & 92,5 & 5 & 2,5 \\
PVA0MMT5.0 & 95,0 & 0 & 5,0 \\
PVA1 MMT5.0 & 94,0 & 1 & 5,0 \\
PVA2 MMT5.0 & 93,0 & 2 & 5,0 \\
PVA3 MMT5.0 & 92,0 & 3 & 5,0 \\
PVA5 MMT5.0 & 90,0 & 5 & 5,0 \\
\hline
\end{tabular}

As misturas foram aquecidas a $90{ }^{\circ} \mathrm{C}$ por $30 \mathrm{~min}$, sob agitação constante de $2000 \mathrm{rpm}$ (agitador magnético Tecnal TE 0851 - São Paulo, Brasi) e $100 \mathrm{~g}$ de cada formulação foram depositadas em placas de acrílico retangulares com área de $200 \mathrm{~cm}^{2}$, as quais permaneceram em estufa com circulação e renovação de ar a $35^{\circ} \mathrm{C}$ por $12 \mathrm{~h}$ para a evaporação do solvente. Após este período, os filmes foram retirados das placas e acondicionados em estufa incubadora para BOD (Tecnal - São Paulo, SP) sob umidade relativa de $60 \%$ e temperatura de 25 ${ }^{\circ} \mathrm{C}$ por $72 \mathrm{~h}$ antes do início das análises.

\section{Caracterização dos filmes}

\section{Espessura}

A espessura dos filmes foi determinada utilizando-se um micrômetro manual ( \pm 0,001 mm) Mitutouyo (São Paulo, Brasil), em dez pontos diferentes de cinco amostras de filmes, considerando-se a espessura do filme como a média entre todas as leituras.

\section{Opacidade}

A medida da opacidade da amostra foi determinada de acordo com Sobral, ${ }^{19}$ utilizando-se colorímetro BYK Gardner (Columbia, EUA).

\section{Microscopia eletrônica de varredura (MEV)}

Analisou-se a microestrutura dos filmes por microscopia eletrônica de varredura, utilizando-se o microscópio eletrônico de varredura FEI Quanta 200 (Oregon - EUA). As amostras foram secas em estufa de circulação de ar (Marconi MA 035) a $105^{\circ} \mathrm{C}$ por $24 \mathrm{~h}$ e, então, fraturadas manualmente e mantidas em dessecadores contendo cloreto de cálcio anidro por 1 semana. Em seguida, as amostras foram recobertas com uma fina camada de ouro e as imagens foram realizadas empregando-se uma voltagem de aceleração de $20 \mathrm{kV}$.

\section{Difração de raios- $X$}

Os difratogramas foram obtidos utilizando-se um difratômetro de Raios-X (X’Pert-Philips). As condições de análise foram: voltagem e corrente - $40 \mathrm{kV}$ e $40 \mathrm{~mA}$, respectivamente; faixa de varredura - $2 \theta$ de 2 a $60^{\circ}$; passo $-0,1^{\circ}$ e velocidade $1 \%$ min, dotado de monocromador de feixe secundário de grafite. A variação dos tamanhos dos cristais foi determinada utilizando-se o PC APD Diffration software. Calculouse o índice de cristalinidade (IC) pela relação: $\mathrm{IC}=\mathrm{A}_{\mathrm{c}} /\left(\mathrm{A}_{\mathrm{c}}+\mathrm{A}_{\mathrm{a}}\right)$, onde $\mathrm{A}_{\mathrm{c}}$ corresponde à área da região cristalina $\mathrm{e} \mathrm{A}_{\mathrm{a}}$, à área da região amorfa. ${ }^{4}$ Foi empregado programa computacional Origin 6.0 para o cálculo das áreas dos difratogramas.

\section{Isotermas de sorção}

Os filmes foram desidratados em dessecador por três semanas, na presença de cloreto de cálcio anidro, que mantém a umidade relativa (UR) próxima a 0\%. Após esse período, as amostras foram acondicionadas a $25{ }^{\circ} \mathrm{C}$ em dessecadores herméticos contendo diferentes soluções saturadas de sais: cloreto de lítio (11\% de UR), cloreto de magnésio (33\% de UR), nitrato de magnésio (52\% de UR), cloreto de sódio (75\% de UR), cloreto de bário (90\% de UR) e sulfato de potássio (97\% de UR). As amostras foram pesadas em intervalos de $2 \mathrm{~h}$ até a $12^{\mathrm{a}} \mathrm{h}$ e, a partir deste período, a cada 24 h até atingirem o equilíbrio. Determinou-se então a umidade de equilíbrio das amostras pela secagem em estufa a $105{ }^{\circ} \mathrm{C}$ por $8 \mathrm{~h}$. As isotermas foram obtidas pela plotagem dos dados de umidade atingida pelas amostras no equilíbrio versus a atividade de água $\left(\mathrm{A}_{\mathrm{a}}\right)$ dos filmes armazenados sob diferentes UR $\left(A_{a}=U R / 100\right)$.

\section{Permeabilidade ao vapor de água (PVA)}

A permeabilidade ao vapor de água foi determinada por método gravimétrico a $25{ }^{\circ} \mathrm{C}$, de acordo com a Norma ASTM E-96-00. ${ }^{20}$ Amostras de cada formulação foram seladas com graxa de silicone em células de permeação de alumínio com $60 \mathrm{~cm}$ de diâmetro, contendo cloreto de cálcio anidro (dessecante). Essas células foram acondicionadas em dessecadores a $25^{\circ} \mathrm{C}$ e $75 \%$ UR. A partir do ganho de peso da cápsula, medido em intervalos de $5 \mathrm{~h}$, durante $24 \mathrm{~h}$, foi possível determinar o vapor de água transferido através do filme. As análises foram realizadas em triplicata.

\section{Propriedades mecânicas de tração}

Os testes de tração foram realizados em um Texturômetro TA.XT2 $i$ (Stable Micro Systems) acoplado de programa computacional Texture Expert 1.15. Amostras dos filmes (25 x $100 \mathrm{~mm})$ foram tracionados a uma velocidade de $500 \mathrm{~mm} / \mathrm{min}$, partindo de uma separação inicial das garras de $50 \mathrm{~mm} .{ }^{21}$ As propriedades mecânicas obtidas nesses testes foram a resistência máxima à tração (RMT) e a elongação na ruptura (E).

\section{Análise estatística}

Para a análise estatística dos resultados obtidos foi empregado o programa computacional Statistica versão 7.0 (Statsoft, Oklahoma, USA). Foi realizado teste de Tukey (nível de significância de 5\%) para comparação de médias. 
Tabela 2. Resultados de espessura, opacidade, permeabilidade ao vapor de água, resistência máxima à tração (RMT) e elongação na ruptura (E) dos filmes de amido de mandioca, PVA e MMT-Na

\begin{tabular}{|c|c|c|c|c|c|}
\hline Formulações & Espessura (mm) & Opacidade aparente & $\begin{array}{l}\text { Permeabilidade ao vapor } \\
\text { de água } \times 10^{10}(\mathrm{~g} / \mathrm{m} \mathrm{s} \mathrm{Pa})\end{array}$ & $\mathrm{RMT}(\mathrm{MPa})$ & $\mathrm{E}(\%)$ \\
\hline PVA0 & 0,134 & $2,30 \pm 0,28 \mathrm{a}$ & $2,91 \pm 0,31 \mathrm{a}$ & $33 \pm 3 \mathrm{e}$ & $52 \pm 2 b$ \\
\hline PVA1 & 0,132 & $2,4 \pm 0,68 \mathrm{a}$ & $2,21 \pm 0,33 b$ & $58 \pm 5 d$ & $60 \pm 1 \mathrm{a}$ \\
\hline PVA2 & 0,138 & $2,55 \pm 0,58 \mathrm{a}$ & $2,52 \pm 0,15 b$ & $58 \pm 3 d$ & $59 \pm 2 a$ \\
\hline PVA3 & 0,141 & $2,56 \pm 0,16 \mathrm{a}$ & $2,35 \pm 0,17 \mathrm{~b}$ & $157 \pm 9 c$ & $58 \pm 2 a$ \\
\hline PVA5 & 0,138 & $2,36 \pm 0,76 \mathrm{a}$ & $2,44 \pm 0,26 b$ & $204 \pm 14 b$ & $61 \pm 1 \mathrm{a}$ \\
\hline PVA0-MMT2.5 & 0,130 & $1,89 \pm 0,27 \mathrm{a}$ & $1,32 \pm 0,56 \mathrm{c}$ & $239 \pm 21 \mathrm{a}$ & $35 \pm 3 c$ \\
\hline PVA1-MMT2.5 & 0,138 & $1,97 \pm 0,27 \mathrm{a}$ & $1,15 \pm 0,11 \mathrm{c}$ & $269 \pm 19 a$ & $27 \pm 2 d$ \\
\hline PVA2-MMT2.5 & 0,131 & $1,99 \pm 0,19 \mathrm{a}$ & $1,42 \pm 0,23 \mathrm{c}$ & $246 \pm 27 \mathrm{a}$ & $37 \pm 3 c$ \\
\hline PVA3-MMT2.5 & 0,130 & $1,88 \pm 0,13 \mathrm{a}$ & $1,53 \pm 0,27 \mathrm{c}$ & $229 \pm 24 a$ & $36 \pm 2 c$ \\
\hline PVA5-MMT2.5 & 0,142 & $1,94 \pm 0,14 \mathrm{a}$ & $1,22 \pm 0,21 \mathrm{c}$ & $267 \pm 28 \mathrm{a}$ & $35 \pm 3 c$ \\
\hline PVA0-MMT5.0 & 0,138 & $1,87 \pm 0,14 \mathrm{a}$ & $1,37 \pm 0,56 \mathrm{c}$ & $245 \pm 25 a$ & $25 \pm 4 d$ \\
\hline PVA1-MMT5.0 & 0,140 & $1,77 \pm 0,31 \mathrm{a}$ & $1,41 \pm 0,02 \mathrm{c}$ & $236 \pm 20 a$ & $37 \pm 3 c$ \\
\hline PVA2-MMT5.0 & 0,141 & $2,01 \pm 0,21 \mathrm{a}$ & $1,12 \pm 0,02 \mathrm{c}$ & $266 \pm 26 a$ & $34 \pm 2 c$ \\
\hline PVA3-MMT5.0 & 0,140 & $1,98 \pm 0,27 \mathrm{a}$ & $1,09 \pm 0,51 \mathrm{c}$ & $216 \pm 31 \mathrm{a}$ & $37 \pm 4 c$ \\
\hline PVA5-MMT5.0 & 0,140 & $1,76 \pm 0,32 \mathrm{a}$ & $1,11 \pm 0,10 \mathrm{c}$ & $260 \pm 33 a$ & $38 \pm 3 c$ \\
\hline
\end{tabular}

a - Valores seguidos de letras diferentes, na mesma coluna, diferem significativamente pelo teste de Tukey (p $\leq 0,05)$. Os resultados estão expressos como média \pm desvio padrão.

\section{RESULTADOS E DISCUSSÃO}

\section{Espessura e opacidade}

Todos os filmes produzidos se mostraram homogêneos, contínuos, sem rachaduras ou poros, podendo ser facilmente removidos das placas. A espessura dos filmes variou de 0,130 a 0,142 mm (Tabela 2) e o tipo de formulação não afetou esta característica dos filmes, uma vez que a porcentagem de sólidos foi mantida constante nas diferentes formulações, levando à adição da mesma quantidade de sólidos em cada placa.

Todas as formulações se mostraram transparentes, o que pode ser confirmado pelos resultados de opacidade (Tabela 2), que variaram de 2,36 a 1,76, e embora a adição de PVA ou nanoargila não tenha afetado de forma significativa (teste de Tukey, $\mathrm{p} \leq 0,05$ ) esta propriedade, a adição de nanoargila levou a uma tendência de queda da opacidade, o que pode ser um bom indicativo de compatibilidade entre os componentes da formulação.

\section{Microscopia eletrônica de varredura}

As micrografias das fraturas dos filmes (Figura 1) revelaram a formação de filmes com estruturas compactas e bastante homogêneas, características de filmes de amido e glicerol produzidos por casting, ${ }^{2}$ no entanto, pode-se perceber nos filmes produzidos com $5 \mathrm{~g}$ de MMT-Na/100 g (Figuras $1 \mathrm{~b}$ e c), com e sem adição de PVA, algumas rachaduras. A MMT-Na atua como agente de reforço para a matriz de amido e a adição nesta concentração pode ter levado à formação de estruturas mais resistentes e rígidas, causando as rachaduras observadas (setas 1 e 2). No entanto, pode-se observar que as rachaduras foram mais evidentes nas amostras produzidas sem o PVA, apenas com a adição de MMT-Na (Figura 1b); isto pode ser explicado pela boa capacidade de interação relatada entre o amido e o PVA, que quando empregados em misturas interagem através de pontes de hidrogênio, levando à formação de matrizes poliméricas homogêneas. ${ }^{10-13}$

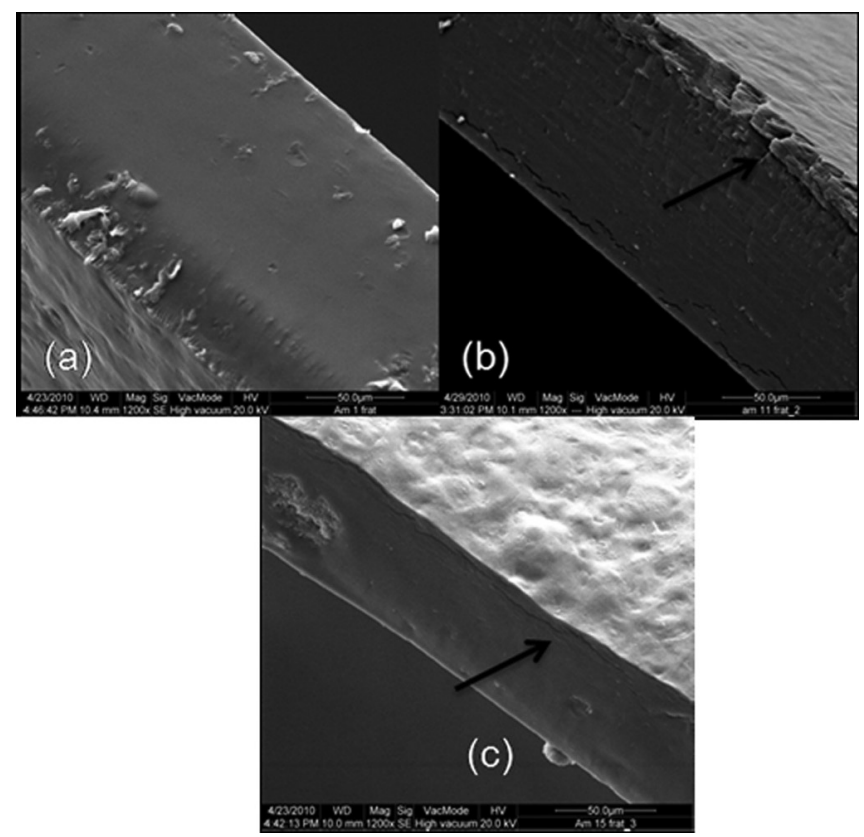

Figura 1. Microscopia eletrônica de varredura das fraturas dos filmes produzidos com as seguintes formulações: (a) PVA0; (b) PVAOMMT5.0; (c) PVA5MMT5.0

Na análise da superfície dos filmes (Figura 2), observou-se uma superfície lisa e homogênea nos filmes produzidos com amido, PVA e MMT-Na, o que é um indicativo da compatibilidade entre os três componentes da formulação. Wilhelm et al., ${ }^{7}$ trabalhando com filmes amido-nanoargila, observaram através de MEV com aumento de 2000 vezes, que as estruturas nanométricas estavam homogeneamente dispersas na matriz, sem a formação de aglomerados, como nos filmes produzidos neste trabalho com amido-PVA-MMT.

Aplicou-se um aumento maior na imagem da fratura (20.000 X) da amostra contendo $3 \mathrm{~g}$ de PVA/100 g de sólidos e $5 \mathrm{~g}$ de MMT- 


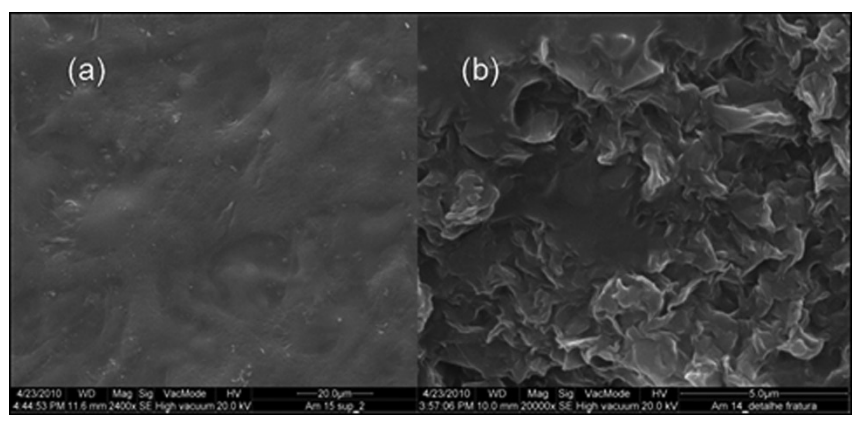

Figura 2. Microscopia eletrônica de varredura das superfícies dos filmes produzidos com as seguintes formulações: (a) PVA5MMT5.0; (b) PVA3MMT5.0

$\mathrm{Na} / 100 \mathrm{~g}$ de sólidos (Figura 2b) e pode-se perceber a formação de uma matriz de amido gelatinizado, no entanto com aspecto uniforme, sem a presença de aglomerados de nanoargilas ou separação de fases.

\section{Difração de raios-X}

Os difratogramas dos filmes de amido de mandioca, PVA e MMTNa estão apresentados na Figura 3. De acordo com alguns autores, ${ }^{22,23}$ o amido de mandioca apresenta cristalinidade tipo $\mathrm{C}$ (combinação do tipo $\mathrm{A}+\mathrm{B}$ ), tendo picos próximos de $2 \theta=15,3^{\circ} ; 17,3^{\circ} ; 18,3^{\circ}$, $22,0^{\circ}$ e $23,5^{\circ}$. Pode-se observar na Figura 3 a presença destes picos no difratograma referente ao amido de mandioca nativo, e pode-se ainda observar que estes picos desapareceram nos filmes, já que o amido sofreu gelatinização durante o processamento das mesmas, com consequente desestruturação das suas regiões cristalinas.
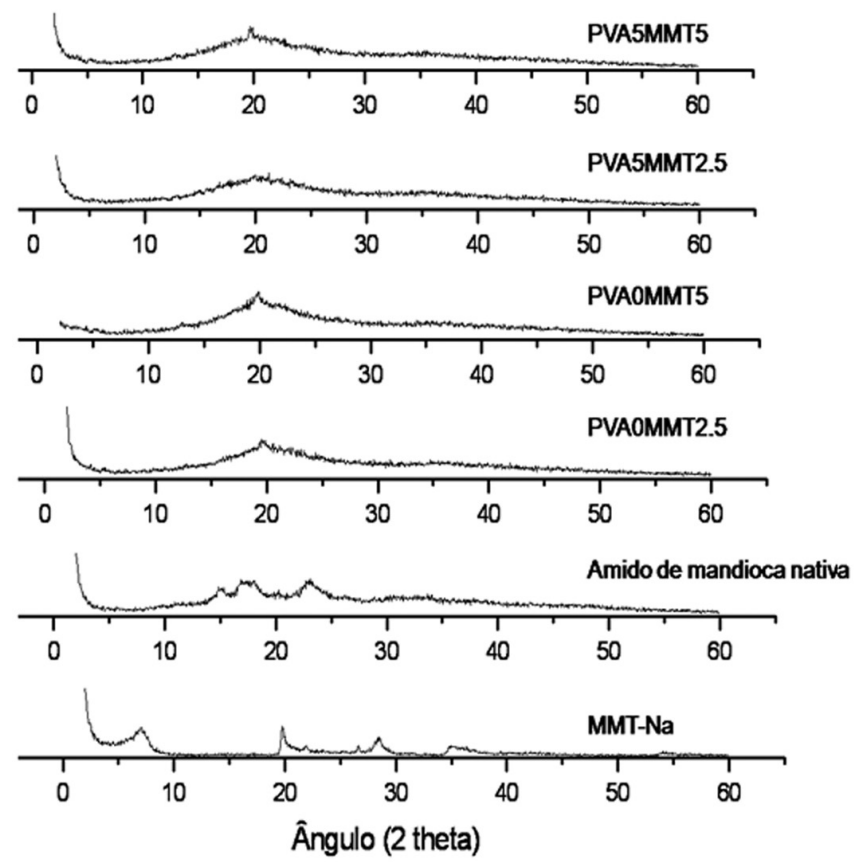

Figura 3. Difratogramas dos filmes à base de amido de mandioca, PVA $e$ $M M T-N a$

De acordo com van Soest e Vliegenthart, ${ }^{24}$ a cristalinidade de uma amostra de um material termoplástico de amido pode ser residual e ocorrer em decorrência de um processamento insuficiente para destruir a sua estrutura cristalina, ou ainda, pode ser induzida pelo processo e estar associada à recristalização da amilose. Provavelmente, o pico observado em $2 \theta=20^{\circ}$ (Figura 3 ) é decorrente do processamento (cristais do tipo $\mathrm{V}_{\mathrm{H}}$ ), característico de materiais processados em temperaturas abaixo de $180{ }^{\circ} \mathrm{C}$ e teores de umidade maiores que $10 \%,{ }^{25}$ como os filmes produzidos neste trabalho.

O álcool polivínilico apresenta um pico importante em $2 \theta=20^{\circ}, 25$ mas neste trabalho, como este pico aconteceu nas amostras que não continham o PVA na mesma intensidade que as amostras que continham, o mesmo foi considerado como característico da cristalinidade do tipo $\mathrm{V}_{\mathrm{H}}$, como discutido acima.

A MMT-Na (Closite ${ }^{\circledR}$-Na) apresenta principal pico de difração em $2 \theta=7,3-7,96^{\circ},{ }^{8,26,27}$ valores similares aos observados neste trabalho, com pico em $2 \theta=7,1^{\circ}$ (Figura 3 ). Pode-se observar (Figura 3 ) que para todos os filmes produzidos com a nanoargila não houve a formação de picos característicos próximos de $2 \theta=7,1^{\circ}$ e nem picos entre $2 \theta$ $=4^{\circ}$ e $2 \theta=10^{\circ}$, sugerindo que houve esfoliação total das lamelas da nanoargila na matriz polimérica. Em nanocompósitos de amido e MMT-Na, quando as lamelas das nanoargilas estão intercaladas, os picos referentes à nanoargila são deslocados para ângulos menores e aparecem em $2 \theta=4-8^{\circ},,^{17,28}$ o que não foi observado neste trabalho.

Provavelmente, o processo de produção das bandejas favoreceu a esfoliação, já que os materiais foram homogeneizados por $30 \mathrm{~min}$ sob aquecimento e agitação mecânica. Chaudhary ${ }^{29}$ relata que a presença de água no processo de produção de nanocompósitos melhora a dispersão das nanoargilas na matriz polimérica.

Os índices de cristalinidade (IC) das formulações analisadas variaram de 14 a $25 \%$ (Tabela 3 ) e todas as amostras apresentaram uma estrutura semicristalina com predomínio da região amorfa e IC menor que o amido de mandioca nativo (30\%), característico de materiais de amido submetidos a processamento térmico. ${ }^{4,24} \mathrm{As}$ amostras de filmes produzidas com a MMT-Na apresentaram menor IC que as produzidas apenas com PVA e amido e, de acordo com Huang et al., ${ }^{30}$ quando existe interação entre o amido e a MMT-Na, isto interfere na formação de pontes de hidrogênio entre as moléculas de amido, restringindo o processo de recristalização, como observado neste trabalho.

Tabela 3. Índices de cristalinidade de algumas formulações dos filmes de amido de mandioca, PVA e MMT-Na

\begin{tabular}{lc}
\hline Formulações & Índice de cristalinidade $(\%)$ \\
\hline PVA0MMT2.5 & 15 \\
PVA5 MMT2.5 & 23 \\
PVA0MMT5.0 & 14 \\
PVA5 MMT5.0 & 22 \\
Amido de mandioca & 30 \\
\hline
\end{tabular}

\section{Isotermas de sorção de umidade}

De acordo com as isotermas de sorção (Figura 4), a adição de nanoargilas se mostrou eficiente em diminuir a higroscopicidade dos filmes de amido. De forma geral, os filmes com a adição de PVA apresentaram uma elevação mais brusca da sua umidade de equilíbrio a partir da $\mathrm{A}_{\mathrm{a}}$ de 0,47 , enquanto que nos filmes adicionados de MMT$\mathrm{Na}$, independente da sua concentração, o aumento mais brusco na umidade de equilíbrio se deu a partir da $\mathrm{A}_{\mathrm{a}}$ de 0,60 , isto é, os valores de umidade destes filmes ficaram praticamente estáveis entre $\mathrm{A}_{\mathrm{a}}$ de 0,11 e 0,60 . Esta característica de estabilidade é o que se busca quando se trabalha com filmes à base de amido e, de acordo com Almasi et $a l .{ }^{9}$ as interações entre o amido e a MMT são do tipo ligações de hidrogênio entre os grupamentos hidroxila presentes nestes compostos, e estas interações substituem em certo grau as interações que a matriz polimérica faria com a água, diminuindo a difusão da água no sistema e melhorando a resistência à umidade nos nanocompósitos. 

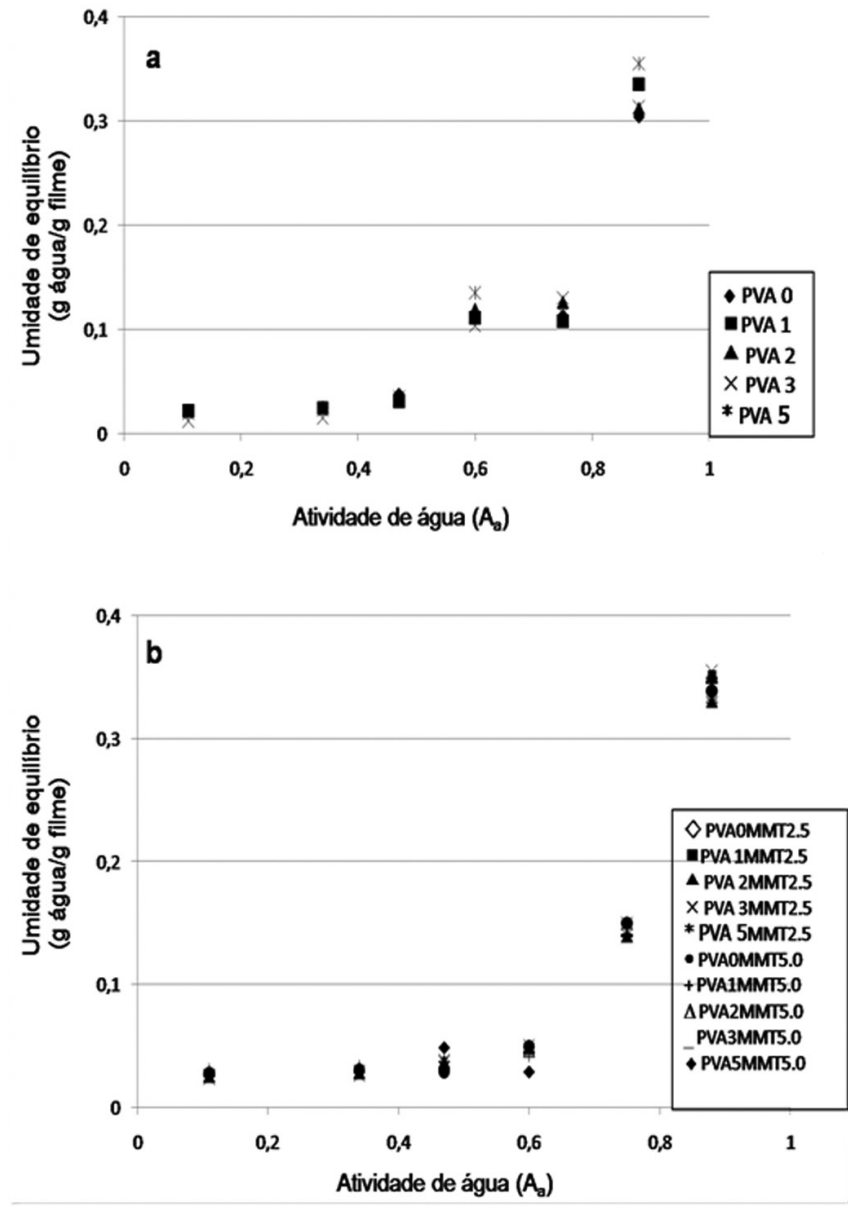

Figura 4. Isotermas de sorção dos filmes à base de a base de amido de mandioca e PVA (a) e à base de amido de mandioca, PVA e MMT-Na

\section{Permeabilidade ao vapor de água}

A permeabilidade ao vapor de água dos filmes variou de 1,09 a $2,91 \times 10^{-10} \mathrm{~g} / \mathrm{msPa}$ (Tabela 2), sendo que o valor da permeabilidade do filme produzido apenas com amido e glicerol (formulação PVA0 - Tabela 2) foi significativamente (teste de Tukey, $\mathrm{p}<0,05$ ) maior que o valor das demais. A adição de PVA levou ao decréscimo significativo da permeabilidade (teste de Tukey, $\mathrm{p}<0,05$ ), no entanto, os menores valores foram obtidos para os filmes adicionados de nanoargilas, independente da concentração de MMT-Na empregada (Tabela 2).

De acordo com Weiss et al., ${ }^{31}$ se as lamelas de argila forem bem distribuídas na matriz polimérica, como é o caso dos filmes produzidos neste trabalho e como foi observado pelos resultados de difração de raios-X discutidos, elas podem criar caminhos tortuosos que dificultam a passagem de vapores, diminuindo a permeabilidade ao vapor de água dos nanocompósitos obtidos.

\section{Propriedades mecânicas}

A resistência máxima à tração (RMT) dos filmes foi afetada significativamente (teste de Tukey, $\mathrm{p}<0,05)$ tanto pela adição do PVA, quanto pela de nanoargilas, que levaram ao aumento da RMT (Tabela 2). As interações entre amido, PVA e a MMT são do tipo ligações de hidrogênio, o que leva a um reforço da matriz polimérica, com aumento da resistência mecânica destes materiais, em especial quando as nanoargilas estão esfoliadas, como os filmes produzidos neste trabalho. Cyras et al. ${ }^{8}$ observaram um aumento significativo da resistência mecânica de filmes de amido de mandioca, elaborados por casting e plastificados com glicerol e adicionados de MMT-Na.

A elongação das amostras aumentou com a adição de PVA e diminuiu com a adição de nanoargilas (Tabela 2). O decréscimo da flexibilidade de filmes de amido com a adição de MMT foi observada por outros autores, ${ }^{8,9}$ que relataram que o efeito reforçador da nanoargila na estrutura da matriz de amido muitas vezes leva ao aumento da rigidez destes materiais, em função do maior número de ligações de hidrogênio formadas entre as hidroxilas do amido e da MMT. Estes resultados de propriedades mecânicas, com aumento da RMT e decréscimo da elongação, estão de acordo com os resultados obtidos nas análises de microscopia eletrônica e difração de raios$\mathrm{X}$, com filmes de superfície homogênea, onde os três componentes interagiram, formando uma estrutura esfoliada quando a MMT-Na foi adicionada.

\section{CONCLUSÕES}

Todos os filmes produzidos se mostraram homogêneos, contínuos, sem rachaduras ou poros. A introdução de PVA à matriz de amido, mesmo em baixas concentrações (1 a 5\%), levou à formação de filmes menos permeáveis ao vapor de água e com melhores propriedades mecânicas. A adição da MMT, que se mostrou esfoliada nos filmes produzidos, originou filmes mais estáveis às diferentes umidades relativas de armazenamento, com menor permeabilidade ao vapor de água e mais rígidos. Filmes produzidos com a combinação de amido, PVA e MMT podem ser uma alternativa para a produção de filmes biodegradáveis com melhores propriedades físico-químicas.

\section{AGRADECIMENTOS}

Ao CNPq (Projeto 577146/2008-4) pelo auxílio financeiro e pela bolsa de Iniciação Científica e à CAPES pela bolsa de Mestrado.

\section{REFERÊNCIAS}

1. Mali, S.; Sakanaka, L.; Yamashita, F.; Grossmann, M. V. E.; Carbohydr. Polym. 2005, 60, 283.

2. Mali, S.; Grossmann, M. V. E.; García, M. A.; Martino, M. M.; Zaritzky, N. E.; Carbohydr. Polym. 2002, 50, 379.

3. Salgado, P. R.; Schmidt, V. C.; Ortiz, S. E. M.; Mauri, A. N.; Laurindo, J. B.; J Food Eng. 2008, 85, 435.

4. Müller, C.; Yamashita, F.; Laurindo, J. B.; Food Hydrocolloid 2009, 23, 1328.

5. Müller, C.; Yamashita, F.; Laurindo, J. B.; Carbohydr. Polym. 2008, 72, 82.

6. Park, H. W.; Lee, W. K.; Park, C. Y.; Cho, W. J.; Ha, C. S.; J. Mater. Sci. 2003, 38, 909 .

7. Wilhelm, H. M.; Sierakowski, M. R.; Souza, G. P.; Wypych, F.; Carbohydr. Polym. 2003, 52, 101.

8. Cyras, V. P.; Manfredi, L. B.; Ton-That, M-T.; Vázquez, A.; Carbohyd. Polym. 2008, 73, 55.

9. Almasi, H.; Ghanbarzadeh, B.; Entezami, A. A.; Int. J. Biol. Macromol. 2010, 46, 1 .

10. Matsumura, S.; Tomizawa, N.; Toki A.; Nishikawa K.; Toshima, K.; Macromolecules 1999, 32, 7753.

11. Aranha, I. B.; Lucae, E. F.; Polímeros 2001, 11, 174.

12. Sudhamani, S. R.; Prasad, M. S.; Sankar, U. K.; Food Hydrocolloid 2003, 17, 245 .

13. Moraes, I. C.; Silva, G. G. D.; Habitante, A. M. Q. B.; Bergo, P. V. A.; Sobral, P. J. A.; Ciênc. Tecnol. Aliment. 2008, 3, 738.

14. Alexandre, M.; Dubois, P.; Mater. Sci. Eng. 2000, $28,1$. 
15. Garcia, E. E. C.; Nanocompósitos: novas opções em materiais de embalagens, Boletim de Tecnologia e Desenvolvimento de Embalagens, ITAL: Campinas, 2003.

16. Coelho, A. C. V.; Souza Santos, P.; Santos, H. S.; Quim. Nova 2007, 30, 1282.

17. Kampeerapappun, P.; Ahtong, D.; Pentrakoon, D.; Srikulkit, K.; Carbohydr. Polym. 2007, 67, 155.

18. Chiou, B. S.; Yee, E.; Glenn, G. M.; Orts, W. J.; Carbohydr. Polym. 2005, 59, 467.

19. Sobral, P. J. A.; Ciência e Engenharia 1999, 8, 60.

20. ASTM - American Society for Testing and Materials - E-96-00; Annual Book of ASTM Standards, ASTM: Philadelphia, 2000.

21. ASTM - American Society for Testing and Materials - D-882-02; Annual Book of ASTM Standards, ASTM: Philadelphia, 2002.

22. Zobel, H. F.; Starch/Starke 1998, 40, 44.
23. Huang, J.; Schols, H. A.; van Soest, J. J. G.; Jin, Z.; Sulmann, E.; Voragen, A. G. J.; Food Chem. 2007, 101, 1338.

24. van Soest, J. J. G.; Vliegenthart, J. F. G.; Trends Biotechnol. 1997, 15, 208.

25. Ricciardi, R.; D’Errico, G.; Auriemma, F.; Ducouret, G.; Tedeschi, A. M.; Rosa, C.; Lauprêtre, F.; Lafuma, F.; Macromolecules 2005, 38, 6629.

26. Chung, Y.; Ansari, S.; Estevez, L.; Hayrapetyan, S.; Giannelis, E. P.; Lai, H. M.; Carbohydr. Polym. 2010, 79, 391 .

27. Majdzadeh-Ardakani, K.; Navarchian, A. H.; Sadeghi, F.; Carbohydr. Polym. 2010, 79, 547.

28. Chen, B.; Evans, J. R. G.; Carbohydr. Polym. 2005, 61, 455.

29. Chaudhary, D. S.; J. Polym. Sci. 2008, 46, 979.

30. Huang, M. F.; Yu, J. G.; Ma, X. F.; Polymer 2004, 45, 7017.

31. Weiss, J.; Takhistov, P.; Mcclements, D. J.; J. Food Sci. 2006, 71, 107. 